\title{
SUBCLINICAL BLOOD PRESSURE ALTERATIONS ARE RELATED TO PROINFLAMATTORY MARKERS IN TYPE 1 DIABETES MELLITUS
}

FJ. Vilchez López ${ }^{1}$, I. Mateo Gavira ${ }^{1}$, A. Montero Galván ${ }^{1}$, F. Visiedo García ${ }^{2}$, MV García Palacios ${ }^{3}$, L. Larrán Escandón ${ }^{1}$, M. Aguilar Diosdado ${ }^{1}$.

1. Endocrinology, Puerta del Mar Hospital, Cádiz, Spain.

2. Investigation Unit of the Hospital Puerta del Mar, Cádiz, Spain.

3. Preventive Medicine and Public Health, Cádiz, Spain.

\section{INTRODUCTION}

The main objective is to evaluate the relationship between precocious subclinical hypertension and inflammatory and endothelial dysfunction markers in normotensive and normoalbuminuric patients with type 1 diabetes.

\section{METHODS}

We designed an observational transversal study of 85 patients, clinically normotensive and without microalbuminuria. Ambulatory blood pressure monitoring (abpm) was performed over $24 \mathrm{~h}$ and subclinical hypertension was considered if: 1 ) mean systolic pressure (sbp) was greater than 130 $\mathrm{mmHg}$ in the 24 hours and daytime periods and greater than $120 \mathrm{mmHg}$ in the nighttime period and/or mean diastolic pressure (dbp) greater than $80 \mathrm{mmHg}$ or $70 \mathrm{mmHg}$ in the same periods respectively, and/or 2) more than $50 \%$ of the readings were higher than the defined previous criteria. Non dipper pattern was defined as nocturnal $\mathrm{sbp}$ or $\mathrm{dbp}<10 \%$ relative to the diurnal mean value. We analyzed the relationship between the blood pressure alterations detected by abpm and inflammatory cytokines (IL-6, TNF- $\alpha$, VEGF) and markers of endothelial damage (VCAM, ICAM and PAI).

\section{RESULTS}

Of the 85 patients included in the analysis, $55,3 \%$ (n:47) were women with an average age of $27,9 \pm 6.1$ years and a length of disease of $12,3 \pm 6.5$ years. $31.8 \%$ presented pathological mean blood pressure parameters in some of the periods. VEGF levels were significantly higher in patients with diurnal blood pressure alterations relative to normotensive patients (table 1). In addition, VEGF levels showed a significant correlation with mean daytime and 24h blood pressure parameters (figure 1 and 2). IL6 levels showed as a risk factor in patients diagnosed with subclinical hypertension (table 2). There were no modifications in the level of markers of endothelial damage.

Table 1. Cytokines and markers of endothelial function damage measured

\begin{tabular}{|c|c|c|c|c|c|c|c|c|c|}
\hline \multirow[b]{2}{*}{ Variable } & \multicolumn{3}{|c|}{ SBP or DBP $24 h$} & \multicolumn{3}{|c|}{ SBP or DBP active period } & \multicolumn{3}{|c|}{ SBP or DBP repose period } \\
\hline & $\begin{array}{l}\text { Pathologic } \\
(n=16)\end{array}$ & $\begin{array}{c}\text { Normal } \\
(n=69)\end{array}$ & p & $\begin{array}{l}\text { Pathologic } \\
\qquad(n=27)\end{array}$ & $\begin{array}{l}\text { Normal } \\
(n=58)\end{array}$ & p & $\begin{array}{l}\text { Pathologic } \\
(n=19)\end{array}$ & $\begin{array}{l}\text { Normal } \\
(n=66)\end{array}$ & p \\
\hline $\begin{array}{l}\text { Seru } \\
\mathrm{pg} / \mathrm{m}\end{array}$ & $\begin{array}{l}3.18 \\
.82-5.43)\end{array}$ & $\begin{array}{c}2.68 \\
(1.58-3.53)\end{array}$ & 0.196 & $\begin{array}{c}3.48 \\
(2.07-5.64)\end{array}$ & $\begin{array}{c}2.29 \\
(1.54-3.29)\end{array}$ & 0.016 & $\begin{array}{c}2.07 \\
(1.52-3.56)\end{array}$ & $\begin{array}{c}2.81 \\
(1.66-3.95)\end{array}$ & 0.501 \\
\hline $\begin{array}{l}\text { Serum TNF- } \alpha \\
\mathrm{pg} / \mathrm{mL}\end{array}$ & $\begin{array}{l}2.61 \\
.43-5.82)\end{array}$ & $\begin{array}{c}2.57 \\
(1.72-4.9)\end{array}$ & 0.836 & $\begin{array}{c}2.45 \\
(0.37-6.37)\end{array}$ & $\begin{array}{c}2.61 \\
1.72-5.11)\end{array}$ & 0.759 & $\begin{array}{c}2.16 \\
(0.31-4.66)\end{array}$ & $\begin{array}{c}2.61 \\
(1.72-5.82)\end{array}$ & 0.405 \\
\hline $\begin{array}{l}\text { Serum VEGF; } \\
\mathrm{pg} / \mathrm{mL}\end{array}$ & $\begin{array}{c}112.33 \\
3.83-219.86)\end{array}$ & \begin{tabular}{|c|}
74.57 \\
$(40.90-128.14)$
\end{tabular} & 0.105 & $\begin{array}{c}112.33 \\
(72.87-213.53)\end{array}$ & \begin{tabular}{|c|}
71.03 \\
$(37.71-107.92)$
\end{tabular} & 0.007 & $\begin{array}{c}85.25 \\
(64.56-140.81)\end{array}$ & \begin{tabular}{|c|}
75.34 \\
$(39.69-132.68)$
\end{tabular} & 0.439 \\
\hline \begin{tabular}{|} 
Plasma sICAM- \\
$1 ; \mathrm{ng} / \mathrm{mL}$
\end{tabular} & $\begin{array}{c}40.65 \\
(28.07-96.12)\end{array}$ & \begin{tabular}{|c|}
56.63 \\
$(38.53-78.65)$
\end{tabular} & 0.256 & $\begin{array}{c}51.45 \\
(37.49-100.29)\end{array}$ & \begin{tabular}{|c|}
56.41 \\
$(35.71-72.83)$
\end{tabular} & 0.600 & $\begin{array}{c}64.12 \\
(41.41-92.22)\end{array}$ & \begin{tabular}{|c|}
53.51 \\
$(33.96-79.21)$
\end{tabular} & 0.390 \\
\hline \begin{tabular}{|l} 
Plasma sVCAM- \\
$1 ; \mathrm{ng} / \mathrm{mL}$
\end{tabular} & $\begin{array}{c}68.66 \\
(24.48-111.01)\end{array}$ & \begin{tabular}{|c|}
58.41 \\
$(37.17-77.86)$
\end{tabular} & 0.617 & $\begin{array}{c}67.83 \\
(41.25-112.22)\end{array}$ & \begin{tabular}{|c|}
52.36 \\
$(35.16-73.59)$
\end{tabular} & 0.073 & $\begin{array}{c}67.83 \\
(36.27-112.22)\end{array}$ & \begin{tabular}{|c|}
55.88 \\
$(37.39-80.64)$
\end{tabular} & 0.359 \\
\hline $\begin{array}{l}\text { Plasma PAI-1; } \\
\mathrm{ng} / \mathrm{mL}\end{array}$ & $\begin{array}{c}46.22 \\
(26.82-190.86)\end{array}$ & \begin{tabular}{|c|}
73.53 \\
$(42.33-150.19)$
\end{tabular} & 0.415 & $\begin{array}{c}92.58 \\
(39.11-26.77)\end{array}$ & $\begin{array}{c}65.42 \\
(41.39-101.81)\end{array}$ & 0.162 & $\begin{array}{c}83.53 \\
(39.11-214.78)\end{array}$ & $\begin{array}{c}66.99 \\
(40.98-142.45)\end{array}$ & 0.480 \\
\hline
\end{tabular}

Figure 1. Correlation between diurnal activity systolic blood pressure and VEGF level

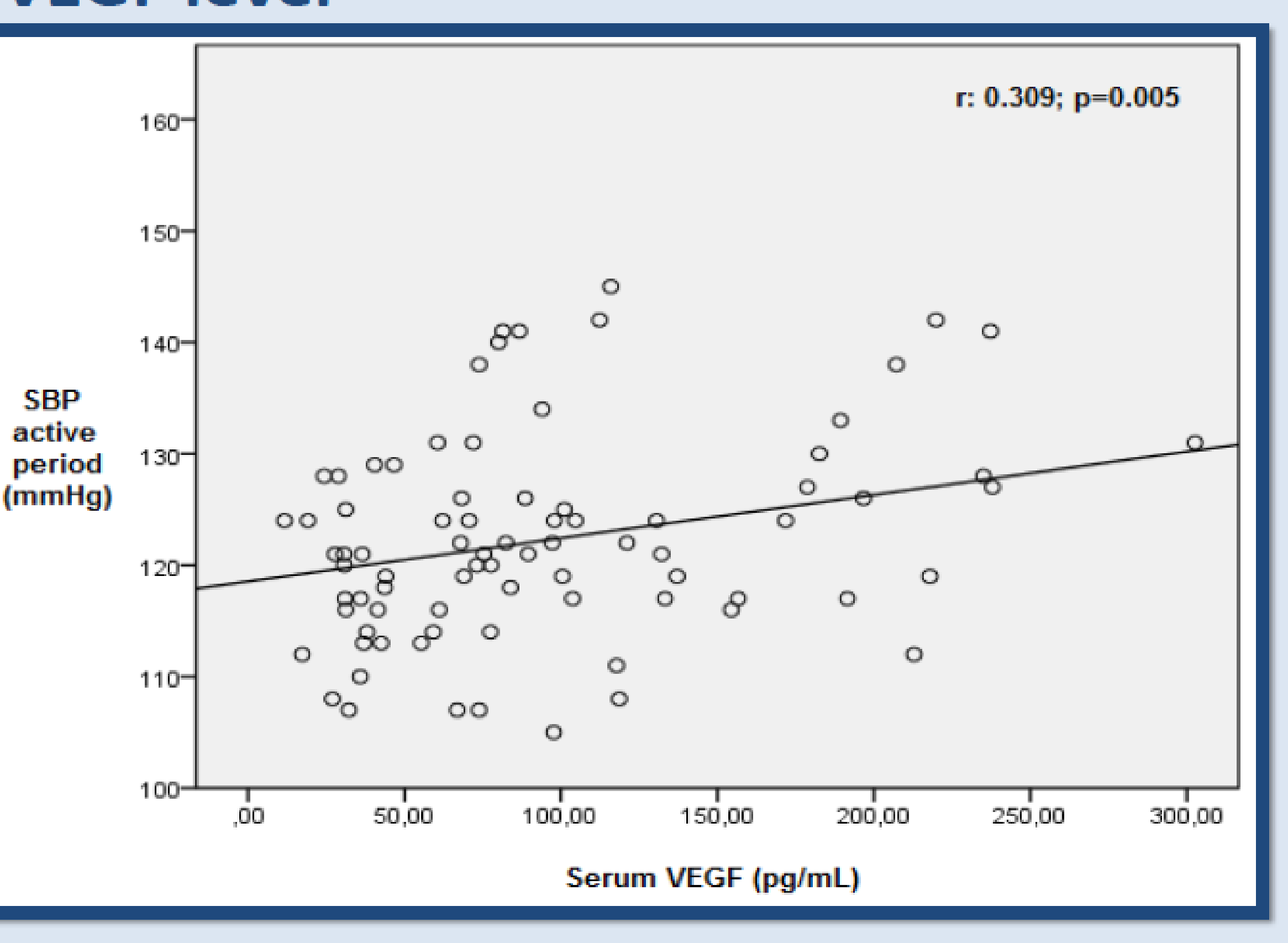

Table 2. Binary logistic regression model for the dependent variable BP pathological in the active period

\begin{tabular}{|l|c|c|c|}
\hline \multicolumn{1}{|c|}{ Variable } & OR & $95 \% \mathrm{Cl}$ & $\mathbf{p}$ \\
\hline HDL-cholesterol & 0.951 & $0.909-0.994$ & $\mathbf{0 . 0 2 2}$ \\
\hline IL-6 & 1.406 & $1.050-1.884$ & $\mathbf{0 . 0 2 7}$ \\
\hline Triglycerides & 1.015 & $1.000-1.030$ & 0.050 \\
\hline
\end{tabular}

Table 3. Multiple linear regression model with the dependent variable VEGF

\begin{tabular}{|c|c|c|c|c|}
\hline Variable & $\beta$ & $\begin{array}{c}\beta \\
\text { standardized }\end{array}$ & $95 \% \mathrm{Cl}$ & $p$ \\
\hline $\begin{array}{l}\text { Mean sbp in } \\
\text { active period }\end{array}$ & 2.254 & 0.280 & $0.144-0.870$ & 0.008 \\
\hline Triglycerides & 0.507 & 0.286 & $0.607-3.902$ & 0.007 \\
\hline \multicolumn{3}{|c|}{$\begin{array}{c}\beta: \text { coefficient of regression; } \beta \text { standardized: standard coefficient of } \\
\text { regression; } 95 \% \text { Cl for } \beta \text { : confidence interval for the coefficient of regression. }\end{array}$} \\
\hline
\end{tabular}

Figure 2. Correlation between diurnal activity diastolic blood pressure and VEGF level

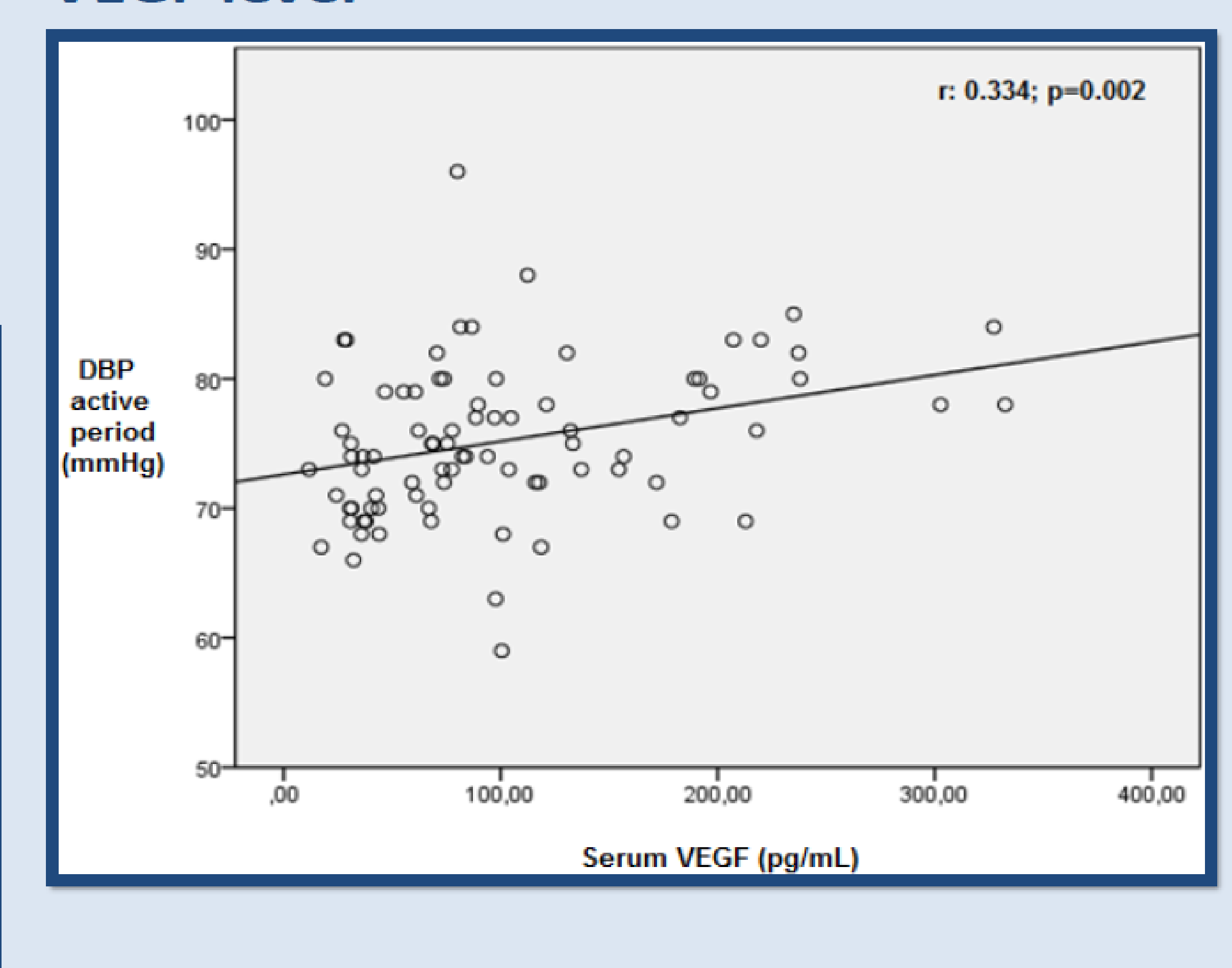

\section{CONCLUSIONS}

An increase in proinflammatory citokines, although not markers of endothelial damage, exists in precocious stages of hypertension in type 1 diabetic patients. 